\title{
Renforcement de la capacité de développement et d'échange des connaissances au Canada : leçons de Youth Excel
}

\author{
B. Riley, Ph. D.; K. Wong, M. Sc.; S. Manske, D. Éd.
}

Cet article a fait l'objet d'une évaluation par les pairs.

\section{Résumé}

Introduction : Youth Excel est une initiative pancanadienne de 3 ans qui a eu comme objectif de favoriser la santé chez les jeunes par le biais de l'amélioration de la capacité de développement et d'échange des connaissances (DEC). La capacité de DEC repose sur un cycle d'amélioration reliant données probantes et interventions. Ses composantes étaient la surveillance locale des comportements des jeunes; l'échange de connaissances; les compétences, les ressources et l'environnement favorable pour l'utilisation des connaissances et enfin l'évaluation.

Méthodologie : Nous avons réalisé des entrevues auprès de membres de Youth Excel relevant de sept équipes provinciales et de deux organisations nationales. Ces entrevues portaient sur les expériences des participants en lien avec le renforcement de la capacité de DEC.

Résultats : L'élément central de la capacité de DEC était constitué par les systèmes de surveillance locale, renforcé par la coordination d'enquêtes au sein des administrations et entre administrations et par le recours à des indicateurs et à des mesures communes. Les échanges de connaissances les plus efficaces étaient ceux qui portaient sur des produits adaptés et qui mettaient à profit les occasions de dialogue et de planification des interventions. La capacité évaluative est demeurée la capacité de DEC la moins élaborée. Le renforcement de la capacité globale de DEC repose sur un dialogue fréquent, des partenariats mutuellement bénéfiques et de la confiance. Il exige également que l'on porte attention au langage, à la vision, au leadership stratégique et au financement.

Conclusion : L'expérience de Youth Excel montre qu'il faut, pour renforcer un système de DEC qui améliore la santé des jeunes, explorer de nouvelles perspectives et obtenir un engagement soutenu de la part des champions ainsi que des organisations concernées.

Mots-clés : développement et échange de connaissances, renforcement des capacités, santé des jeunes, application des données probantes dans la pratique, santé publique fondée sur des données probantes, surveillance locale

\section{Introduction}

La capacité de produire et d'utiliser des données probantes pertinentes et de bonne qualité est fondamentale en santé publique. Quelle que soit la manière dont elles sont décrites, les fonctions essentielles de la santé publique comprennent l'évaluation des profils et des tendances en matière de santé ainsi que l'élaboration, la mise en œuvre et l'évaluation de programmes et de services de santé en partenariat avec les collaborateurs concernés $^{1,2}$. Il a été prouvé que la capacité à exercer ces fonctions est limitée au sein de nombreuses administrations, et on a voulu remédier à cette situation dans le domaine de la santé publique ${ }^{3-6}$.
C'est en réponse à ces besoins qu'une équipe pancanadienne a mis sur pied l'initiative Youth Excel, un programme de 3 ans financé par l'initiative Connaissances et action liées pour une meilleure prévention (COALITION) du Partenariat canadien contre le cancer $^{7}$. L'objectif de l'initiative Youth Excel était de renforcer les capacités à produire et à utiliser des données probantes - appelées capacités de développement et d'échange des connaissances (DEC) - pour améliorer la santé des jeunes Canadiens de 10 à 18 ans. Le projet de DEC a eu comme objectifs d'améliorer le cycle liant données probantes et interventions, notamment par la surveillance locale des comportements des jeunes, de favoriser l'acquisition des compétences, la disponibilité en ressources et l'existence d'un environnement favorable pour l'utilisation et l'échange des connaissances et enfin de mettre en place un processus d'évaluation (détaillé plus bas).

L'évaluation de Youth Excel a fait appel à des méthodes internes mixtes, dont une étude qualitative visant à déterminer les leçons retenues à propos du renforcement de la capacité de DEC. Cet article présente les résultats de cette étude qualitative.

\section{Contexte de la recherche: Youth Excel}

La promotion de la prévention et de la santé auprès de la population, par le biais d'une attention particulière accordée au mode de vie sans tabac ainsi qu'à l'activité physique et à une saine alimentation, est la priorité affirmée du gouvernement et des organisations non-gouvernementales au Canada ${ }^{8}$, aux États-Unis ${ }^{9}$ et à l'échelle mondiale ${ }^{10}$. L'initiative «Youth Health 
Collaborative: 'Excelerating' EVIDENCEinformed ACTION » (c'est-à-dire Youth Excel) a ciblé ces priorités chez les jeunes de 10 à 18 ans. Comme les autres initiatives de COALITION, Youth Excel a eu comme objectif d'accélérer la diffusion et la mise en œuvre des politiques et des pratiques fondées sur des données probantes au moyen de la collaboration entre les différentes administrations et entre les secteurs de la recherche, des politiques et de la pratique. Youth Excel a été axé sur les capacités essentielles à la prise de décisions et à la réalisation d'interventions fondées sur des données probantes. Les origines et la mise sur pied de Youth Excel ayant été déjà décrites de façon détaillée ailleurs $^{11}$, nous n'en offrirons ici qu'un bref aperçu.

Youth Excel a été créé par un groupe de personnes ayant déjà collaboré à d'autres projets et poursuivant un but commun : s'assurer que les priorités, les programmes et les politiques du Canada en matière de santé des jeunes sont fondés sur des données probantes. Des équipes provenant de sept provinces (ColombieBritannique, Alberta, Manitoba, Ontario, Nouveau-Brunswick, Terre-Neuve et Îledu-Prince-Édouard) et de deux organisations nationales - le Consortium conjoint pour les écoles en santé (CCES) et le Centre pour l'avancement de la santé des populations Propel (Propel) — ont imaginé un avenir dans lequel les chefs de file en matière de politiques, de pratiques et de recherche des ordres de gouvernement fédéral, provincial et territorial au Canada se réuniraient régulièrement pour (1) définir conjointement les priorités d’intervention, définir les besoins les plus pressants et les interventions les plus prometteuses; (2) agir sur les priorités; (3) tirer des leçons des mesures prises et évaluer les méthodes prometteuses et (4) améliorer constamment les politiques et les programmes permettant d'améliorer la santé des jeunes. Cette vision de Youth Excel implique l'existence d'un cycle d'amélioration reliant données probantes et interventions. La référence à ce cycle pour Youth Excel est appelée la capacité de DEC. Il intègre quatre composantes : la surveillance locale des comportements des jeunes, l'échange des connaissances, les compétences et les conditions dans les- quelles les données pourront être utilisées ainsi que l'évaluation (figure 1).

Pour renforcer la capacité de DEC, Youth Excel a valorisé les partenariats en cours et en a établi de nouveaux. Les membres travaillaient dans les secteurs de la recherche, des politiques et des pratiques, ainsi que dans les secteurs de la santé et de l'éducation. Certains cumulaient jusqu'à 16 années de collaboration dans le cadre d'autres projets avant de s'engager dans Youth Excel. Notons que le stade de développement et les initiatives liés à la capacité de DEC variaient considérablement entre les sept équipes provinciales participantes (tableau 1). Cette variabilité constituait une " expérience naturelle » qui a guidé les buts et les activités de Youth Excel.

Les membres de Youth Excel ont défini cinq activités principales visant à renforcer la capacité de DEC. La première tirait profit les progrès plus avancés de certaines provinces en matière de DEC : des études de cas comparatives et approfondies sur la capacité de DEC ont été menées dans quatre provinces (Alberta, Manitoba, Nouveau-Brunswick et Île-du-PrinceÉdouard). La deuxième a consisté à organiser des forums nationaux réunissant des intervenants des secteurs des politiques, des pratiques et de la recherche afin de partager les connaissances, d'apprendre et de découvrir de nouvelles façons de renforcer la capacité de DEC. Par exemple, un forum national a visé à partager des leçons retenues grâce aux études de cas provinciales et à donner l'occasion aux membres de planifier les interventions nécessaires au sein des provinces et des territoires. La troisième activité a porté sur l'établissement de forums provinciaux et a donné lieu à l'organisation, en 3 ans, de 15 forums s'appuyant sur les activités et les résultats des forums nationaux. La quatrième activité de Youth Excel a consisté à organiser un programme d'échange des connaissances avec les pairs des différentes provinces ciblant les domaines de la santé et de l'éducation, ainsi que les secteurs de la recherche, des politiques et des pratiques afin de renforcer la collaboration et les apprentissages au sujet de la capacité de DEC au sein de divers groupes. Enfin, Youth Excel a collaboré avec des experts en matière de recherche, de politique et de pratique à l'échelle nationale pour établir un ensemble d'indicateurs et de mesures du tabagisme, de l'activité physique et de la saine alimentation chez les jeunes susceptibles d'être utilisés comme outil de surveillance ou de suivi.

\section{Méthodologie}

Des entrevues téléphoniques ont été réalisées auprès des membres de Youth Excel des 7 équipes provinciales et des 2 organisations nationales. Les 21 participants, dont les commentaires ont été recueillis par l'entremise de 12 entrevues, étaient des chercheurs (12), des praticiens (5) et des décideurs (4). Chaque organisation nationale et chaque équipe provinciale constituait une unité de collecte et d'analyse de données. Les entrevues en groupe ont permis de confronter différents points de vue au sein des organisations et des équipes. Plus du tiers des répondants (8) travaillaient directement à l'échelle locale ou communautaire. La participation

FIGURE 1

Cycle de la capacité de développement et d'échange des connaissances

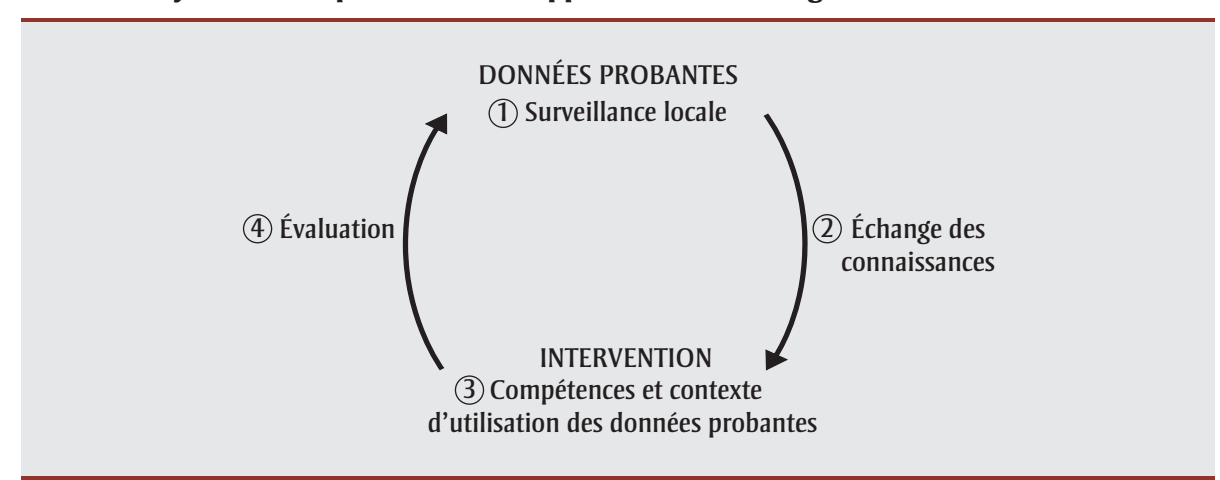


TABLEAU 1

Membres provinciaux et nationaux de Youth Excel et historique de leur collaboration

Partenaires provinciaux (organisation responsable)

Colombie-Britannique (Université de Victoria)

Alberta (Université de l'Alberta)

Manitoba (Action Cancer Manitoba)

Ontario (Santé publique Ontario)

Nouveau-Brunswick (Université du Nouveau-Brunswick)

Terre-Neuve-et-Labrador (Université Memorial)

Île-du-Prince-Édouard (Université de l'île-duPrince-Édouard)

\section{Description des partenaires et historique de leur collaboration}

Les membres de Youth Excel en C.-B. tissent des liens étroits entre la recherche et la pratique. En 2004, un des intervenants clés est passé du secteur gouvernemental (santé) au milieu universitaire. Il a su maintenir des liens étroits avec le secteur gouvernemental par le biais de l'élaboration et de l'évaluation de stratégies gouvernementales telles que le projet Action Schools! $B C$, une initiative provinciale visant à favoriser la mise en œuvre de changements systémiques pour l'atteinte d'un poids santé à l'aide d'approches gilobales de la santé en milieu scolaire et d'un programme d'éducation sur la consommation de boissons sucrées. Les intervenants clés en C.-B. et en Alberta collaborent afin de s'assurer que leurs provinces recueillent des données comparables. Les responsables provinciaux de la C.-B. et de l'Ontario collaborent à la recherche visant à éclairer les décisions en matière de diffusion de l'information.

La Population Health Intervention Research Unit (PHIRU) de l'Université de l'Alberta réalise des études d'intervention au sein de la population en Alberta, à l'î.-P.-É. et en N.É. Par exemple, la PHIRU évalue les investissements de l'Alberta Health and Wellness en matière de saine alimentation et de vie active au moyen de l'enquête REAL Kids menée auprès des élèves de $5^{\mathrm{e}}$ année. Le projet APPLE Schools est un autre projet de collaboration qui permet d'évaluer le modèle global de santé en milieu scolaire. L'initiative EverActive Schools, une initiative globale de santé en milieu scolaire, collabore également avec Youth Excel. La PHIRU et EverActive Schools ont également établi un partenariat avec le gouvernement pour évaluer d'autres modèles de mise en œuvre d'initiatives globales de santé en milieu scolaire.

Le réseau Partners in Planning for Healthy Living (PPHL) du Manitoba assure la surveillance des facteurs de risque locaux ainsi que le développement et l'échange des connaissances pour la planification à l'échelle locale en accordant d'abord la priorité à la santé des jeunes. Fondé en 2006, le PPHL comprend (1) l'ensemble des 11 autorités sanitaires régionales du Manitoba; (2) des organisations non gouvernementales (Alliance pour la prévention des maladies chroniques, Société canadienne du cancer - division Manitoba, Action Cancer Manitoba, Fondation des maladies du cœur du Manitoba); (3) trois ministères provinciaux (Santé et vie saine Manitoba; Éducation, Citoyenneté et Jeunesse Manitoba; Enfants en santé Manitoba); (4) l'organisme Health in Common et (5) les bureaux régionaux de l'Agence de la santé publique du Canada (région du Manitoba et de la Saskatchewan). Le PPHL harmonise les mandats, les priorités, les investissements et les activités entre les diverses organisations en vue de mettre en commun les ressources et de collaborer au soutien de l'utilisation des données probantes pour planifier les interventions liées à la promotion d'un mode de vie sain dans les communautés du Manitoba. Le Manitoba a mené à bien l'enquête sur la santé des jeunes, qui favorise les interventions fondées sur les données recueillies à l'échelle locale.

Les collaborateurs de l'Ontario ont établi de nombreuses interconnexions. Ils ont mené une vaste gamme de projets, comprenant le projet SIPÉSÉ (voir la description dans la section réservée au Centre pour l'avancement de la santé des populations Propel, ci-dessous). Cette collaboration à l'échelle provinciale fait intervenir des responsables de travaux de recherche de Propel et du ministère de la Santé et des Soins de longue durée de l'Ontario (anciennement le ministère de la Promotion de la santé), les directions régionales de la santé publique et Santé publique Ontario (SPO). SPO fournit des conseils de nature scientifique et technique à ceux dont le travail consiste à protéger et à promouvoir la santé des Ontariens. SPO renforce la capacité de DEC par le biais des centres de ressources pour la promotion de la santé en Ontario, effectue des travaux de recherche et d'évaluation liés à la mission et fournit des données probantes susceptibles d'influencer directement les politiques et la pratique.

Le ministère du Mieux-être, de la Culture et du Sport du N.-B., le ministère de l'Éducation et le Groupe de recherche en santé et en éducation (GRSÉ) de l'Université du N.-B. ont uni leurs efforts pour élaborer une stratégie axée sur le bien-être des jeunes du N.-B. Les principaux intervenants ont collaboré pendant 10 ans dans le cadre de la lutte antitabac et ont dirigé la coalition antitabac du N.-B. (y compris les forums jeunesse provinciaux). Les partenaires du N.-B. ont effectué l'évaluation et la promotion de la santé chez les jeunes dans l'ensemble des écoles provinciales suivant des cycles de trois ans à partir de 2006-2007, et ont assuré le soutien du suivi en milieu scolaire afin de définir les priorités, les forces de la communauté et les domaines d'intervention.

Par le biais de l'initiative Healthy Schools Healthy Students Initiative (HSHS), le gouvernement de Terre-Neuve-et-Labrador appuie une approche globale de la santé en milieu scolaire à l'aide d'un comité interministériel (santé, éducation, loisirs, santé environnementale) et d'un comité responsable de HSHS. Le comité provincial responsable de HSHS réunit des chercheurs, des coordonnateurs de la santé en milieu scolaire, des décideurs et des fonctionnaires civils des arrondissements scolaires ainsi que des autorités sanitaires. L'initiative HSHS a appuyé diverses politiques et projets : mise en œuvre de politiques sans tabac dans les établissements scolaires des arrondissements, élaboration de lignes directrices sur l’alimentation en milieu scolaire, élaboration, mise en œuvre et évaluation de projets Active Schools dans tous les arrondissements scolaires et mise en œuvre d'un nouveau programme d'éducation physique.

Depuis 2005, le groupe Comprehensive School Health Research (CSHR) de l'Université de l'î.-P.-É. travaille avec le ministère de l'Éducation et du Développement de la petite enfance de l'Î.-P.-É. à l'amélioration de la santé de la population. Le ministère de l'Éducation de l'Î.-P.-É. a financé trois cycles d'une enquête réalisée dans des écoles de l'Î.-P.-É. à l'aide du projet SIPÉSÉ (voir la description dans la section réservée au Centre pour l'avancement de la santé des populations Propel, ci-dessous) visant à recueillir des données de CSHR sur les élèves. Puisque l'on met désormais l'accent sur l'échangé des connaissances plutôt que sur la collecte de données, les partenaires de l'î.-P.-É. ont établi des liens avec d'autres partenaires de longue date (sur une période pouvant aller jusqu'à 20 ans) comprenant la Société canadienne du cancer - division de l'î.-P.-É., le ministère de la Santé de l'î.-P.-É., les trois commissions scolaires de la province et les écoles de l'î.-P.-É. Ces groupes ont également établi des liens avec des regroupements provinciaux pertinents pour Youth Excel, notamment l'Alliance contre le tabagisme de l'î.-P.-É., l'organisme Recreation PEI et l'Alliance pour une saine alimentation de l'î.-P.-É. 
TABLEAU 1 (SUITE)

Membres provinciaux et nationaux de Youth Excel et historique de leur collaboration

Partenaires nationaux

Consortium conjoint pancanadien pour les écoles en santé (CCES)

Centre pour l'avancement de la santé des populations Propel (Propel)

\section{Description des partenaires et historique de leur collaboration}

Le CCES découle d'un accord conclu en 2005 entre les ministres provinciaux et territoriaux de la Santé et de l'Éducation ainsi que l'Agence de la santé publique du Canada pour assurer le leadership et renforcer la capacité des secteurs de l'éducation et de la santé afin d'améliorer la santé et la réussite des élèves. Le CCES a donné lieu à un effort pangouvernemental sans précédent pour promouvoir la collaboration au sein des administrations et entre celles-ci. Il a entrepris un certain nombre d'activités fondamentales pour la capacité de DEC. Le CCES a tenu des réunions sur la surveillance des données avec des experts invités et des décideurs provenant de diverses régions au Canada afin de faire progresser les questions liées à la collecte et à la surveillance des données concernant le programme global de santé en milieu scolaire. Conjointement avec Propel, il a élaboré et mis à l'essai le Planificateur des écoles en santé, un outil d'évaluation fondé l'approche globale de la santé en milieu scolaire, pour recueillir des données probantes qui orientent la planification et les interventions effectuées par les écoles et leurs partenaires. Le CCES a communiqué de façon proactive avec des organisations nationales clés telles que le Réseau de santé publique et l'Association canadienne des directeurs d'école afin de faciliter l'avancement du programme et d'assurer l'établissement de liens et l'harmonisation des activités.

Propel, une entreprise de collaboration, effectue des recherches, des évaluations et de l'échange de connaissances afin d'accélérer les améliorations en matière de santé des populations. Ses domaines d'intérêt sont la lutte contre le tabagisme, la santé des jeunes, la prévention des maladies chroniques et le développement de la capacité. Propel se spécialise dans l'établissement de liens entre les données probantes et les interventions visant à prévenir le cancer et d'autres maladies chroniques. Propel travaille de façon conjointe avec plus de 200 chefs de file des domaines de la science, des politiques et de la pratique au Canada afin de planifier et de mener des études ainsi que de prendre des mesures en fonction des résultats dans le but d'améliorer les politiques et les programmes et d'orienter les changements. En plus d'élaborer le Planificateur des écoles en santé avec le CCES, Propel a mis au point le Système d'intervention, de planification et d'évaluation de la santé dans les écoles (SIPÉSÉ), qui permet de recueillir de façon efficace des données probantes sur des sujets clés concernant les élèves et de transmettre ces informations aux écoles et à leurs partenaires. Propel s'emploie à mettre en application les données probantes, en particulier en jouant un rôle de rassembleur.

Abréviations : C.-B., Colombie-Britannique; DEC, développement et échange des connaissances; Î.-P.-É., île-du-Prince-Édouard; N.-B., Nouveau-Brunswick; N.-É., Nouvelle-Écosse.

directe à la conception, à la mise en œuvre ou à l'évaluation de l'initiative Youth Excel était le critère principal de sélection des participants aux entrevues, étant donné que ces entrevues visaient à comprendre les atouts et les facteurs favorisant le renforcement de la capacité de DEC ainsi que les obstacles auxquels il avait fait face pendant la période de financement de l'initiative, soit jusqu'en novembre 2011. Ces entrevues, d'environ une heure, ont été menées par deux des auteurs (KW, SM) en novembre et en décembre 2011. Elles ont suivi un guide d'entrevue semi-structurée comprenant 12 questions élaboré par le groupe de travail d'évaluation de Youth Excel Ce guide avait été préalablement envoyé aux participants afin qu'ils recueillent les commentaires d'autres membres de Youth Excel au sein de leur administration.

Les entrevues ont été enregistrées, transcrites et importées dans le logiciel NVivo 9 (QSR International, Doncaster, Australie) puis soumises à trois passages de codification des réponses. Chaque analyse s'articulait autour de l'une des quatre composantes de la capacité de DEC, dans le but de comprendre les éléments essentiels au développement de chacune des composantes de la capacité de DEC.
La première analyse des réponses portait sur le codage, ligne par ligne, du texte des entrevues dans les catégories de composantes de DEC sélectionnées. Cette étape comprenait la segmentation et l'étiquetage du texte de l'entrevue, l'attribution d'une première catégorie de composante de DEC au texte codé, puis l'attribution d'autres catégories pertinentes au texte codé le cas échéant. Après le codage de toutes les entrevues, les codes ont été revus afin de veiller à ce qu'ils appartiennent bien à la catégorie de composante de DEC sélectionnée. La deuxième analyse portait sur le tri des codes dans les catégories au sein de chacune des composantes de DEC.

La troisième analyse de codage a permis de définir les thèmes spécifiques à chaque composante en matière de capacité de DEC. Les critères de définition de ces thèmes étaient soit l'existence de discussions autour de ce thème pour la majorité des participants en lien avec la capacité de DEC, soit la mesure dans laquelle les participants avaient discuté de ce thème dans le cadre des entrevues. En outre, les entrevues au cours desquelles on avait relevé la présence d'un thème (p. ex. partenariats mutuellement bénéfiques, instaurer la confiance, dialogue fréquent, etc.) propre à une composante de DEC ont été systématiquement comparées à celles qui ne présentaient pas ce thème, afin de déterminer si le thème en question était véritablement un élément essentiel. Enfin, les thèmes des quatre composantes de DEC ont été comparés afin d'établir des recoupements (thèmes transversaux) potentiels.

Toutes les méthodes de recherche ont été approuvées par le Bureau de l'éthique en recherche de l'Université de Waterloo. Tous les participants ont donné leur consentement éclairé.

Nous avons vérifié la pertinence de nos constatations de plusieurs façons. L'étude incluait le chercheur principal et le personnel de chaque équipe provinciale et de chaque organisation nationale participant le plus activement à l'initiative Youth Excel. Des entrevues non structurées en petits groupes (2 ou 3 personnes) ont permis de rendre compte de façon efficace de l'étendue et de la profondeur de l'expérience dans chaque ordre de gouvernement. Pour éviter d'introduire un biais dans les réponses, nous nous sommes assurés que l'objet des entrevues et le moment où elles ont été réalisées n'entraient pas en conflit avec une demande de financement en cours de préparation ou d'évaluation. Une version 
préliminaire des résultats a été distribuée à tous les participants, afin qu'ils indiquent si ces résultats étaient en accord avec leur expérience. Enfin, l'analyse des résultats a été confiée à une personne non affiliée à Youth Excel.

L'étude a été délibérément limitée aux membres de Youth Excel pour l'exploration de la capacité de DEC. De fait, les membres du domaine de la recherche étaient plus nombreux à avoir participé que ceux du domaine des politiques et des pratiques, ce qui reflétait les affiliations de l'ensemble de l'équipe de Youth Excel. Par ailleurs, l'expérience des répondants variait en ce qui concerne les quatre composantes de DEC : les résultats pour les deux premières composantes s'appuyaient sur l'expérience directe et approfondie, tandis que les résultats pour la troisième et la quatrième composantes reposaient sur un nombre moindre d'expériences.

\section{Résultats}

Les résultats ont été regroupés en fonction des quatre composantes de capacité de DEC (voir la figure 1) et en fonction de la capacité globale de DEC pour les thèmes recoupant au moins 2 composantes (thèmes transversaux). Les résultats reflètent les expériences des participants en ce qui concerne le renforcement de la capacité de DEC, tant avant que pendant les 3 ans de l'initiative Youth Excel. Pour préserver l'anonymat des répondants, les points de vue des membres exprimés pendant les entrevues ont été cotés selon le secteur auquel ces membres appartenaient $(\mathrm{R}=$ recherche, $\mathrm{P}=$ politiques et $\operatorname{Pr}=$ pratiques) et selon le niveau de compétence administrative dont ils dépendaient (Prov = provinciale; Nat $=$ nationale). Les entrevues s'étant à l'origine déroulées en anglais, il s'agit pour cette version française de traductions.

\section{Composante 1 : Système de surveillance locale}

Les systèmes de surveillance locale recueillent des données sur des sujets prioritaires définis par les intervenants et jugés essentiels pour la capacité de DEC, par exemple le tabagisme, l'activité phy- sique, une saine alimentation et une bonne santé mentale.

La principale différence, c'est que nous avons maintenant des données mesurables dont nous ne disposions pas auparavant. Dans le passé, nous avions des opinions. Nous avions très peu de données à l'échelle nationale. Sans les données probantes, nous ne pouvions pas avoir les conversations que nous avons maintenant avec le gouvernement. Le fait de disposer de ces données provinciales sur les étudiants de notre province m'est utile au niveau de l'école. (R; Prov)

Les thèmes suivants reflètent les observations des répondants à propos des éléments des systèmes de surveillance locale et de la façon de créer des systèmes de ce type dans les différentes administrations.

\section{Partenariats mutuellement bénéfiques}

Les membres de Youth Excel ont souligné l'importance des relations et des réseaux dans l'élaboration d'un système de surveillance locale efficace (p. ex. «L'établissement de relations est l'essence même du développement et de l'échange des connaissances » [R; Prov]). Les membres ont indiqué que l'exploitation des réseaux en place était particulièrement importante, tout comme l'établissement de partenariats avec des " agents de liaison ", c'est-à-dire des personnes ayant des liens avec d'autres chercheurs, praticiens ou décideurs, ainsi qu'avec des " négociateurs ", c'est-à-dire des personnes qui savent comment trouver et mobiliser d'autres intervenants et qui accélèrent le processus d'adhésion des intervenants et d'exploitation des forces complémentaires de chacun.

Les membres de Youth Excel considéraient tous le gouvernement comme un intervenant clé, bien que le rôle qu'ils en attendaient ait différé en fonction de l'ordre de gouvernement concerné. Certains estimaient qu'un outil de collecte de données affichant une mention d'approbation ou un logo du gouvernement serait considéré avec plus de sérieux qu'un outil n'en affichant aucun.
D’après mon expérience personnelle, si je distribue un questionnaire d'enquête qui porte non seulement un logo de projet, mais également les logos du gouvernement de [la province] [...], le questionnaire est considéré avec plus de sérieux. Aussi, lorsque j'ai commencé les travaux de ce contrat avec le gouvernement, un contrat que je considérais comme un partenariat, j'ai particulièrement insisté pour pouvoir afficher le logo du gouvernement sur le questionnaire d'enquête. (R; Prov)

\section{Instaurer la confiance}

L'établissement et le maintien de systèmes de surveillance locale exigent l'instauration d'un climat de confiance entre les différents intervenants. La négociation avec des membres ayant différentes perspectives en matière de recherche, de politiques et de pratiques, et l'établissement d'une vision commune avec ces divers intervenants se sont révélés complexes et parfois difficiles. Pour instaurer la confiance, les membres avaient besoin d'un environnement où l'on ne porte pas de jugement, d'une volonté et d'une faculté d'adaptation, de transparence et d'un engagement indéfectible à l'égard de la vision et des valeurs établies par les membres de l'équipe.

Je crois que cela témoigne de nos convictions profondes à l'égard de l'instauration de la confiance et de l'établissement de relations pour former de véritables partenariats et du fait qu'il faut se donner le temps nécessaire pour y arriver. (R; Prov)

\section{Dialogue fréquent}

Les membres de Youth Excel jugeaient que les réunions régulières et les réunions spécifiques en personne avec un groupe varié étaient essentielles. Ils estimaient qu'il était également important que de telles réunions comprennent des représentants ne faisant pas partie des secteurs de la santé et de l'éducation mais qui étaient conscients de la valeur ajoutée et de l'application pratique d'un système de surveillance. Les membres ont signalé que des réunions fréquentes facilitaient la tenue de "conversations démocratiques", 
la mobilisation des partenaires internes et externes et la résolution de problèmes liés à des questions complexes telles que la coordination d'enquêtes multiples.

\section{Coordination des enquêtes}

Aux échelons national, provincial et local, de nombreuses enquêtes permettent de recueillir des données sur des sujets particuliers et communs liés à la santé des jeunes. Les membres de Youth Excel ont souligné les avantages de la coordination des enquêtes au sein de toutes les administrations, ce qui permettrait de mieux utiliser les ressources et de réduire le fardeau pour les répondants et les organisations :

[...] des discussions se tiennent dans l'ensemble de la province sur la façon dont nous pouvons, en premier lieu, utiliser les enquêtes existantes et les coordonner et, en deuxième lieu, combler les lacunes qui existent à l'heure actuelle, en particulier pour ce qui touche les questions de l'obésité, de l'activité physique et de l'alimentation saine chez les enfants. (R; Prov)

Les membres ont également souligné la nécessité de bien comprendre les différents contextes et la valeur de rassembler les intervenants clés qui sont en mesure d'assurer cette coordination.

\section{Indicateurs et mesures de base}

De nombreux répondants ont exprimé le besoin d'utiliser des questions uniformes dans les différents outils de surveillance ou de suivi au fil du temps pour faciliter la réalisation de comparaisons significatives entre les contextes et les groupes démographiques. L'aspect le plus intéressant des indicateurs et des mesures élaborés par Youth Excel était le processus consensuel entourant la recherche, les politiques et les pratiques ainsi que leur souplesse d'utilisation. Par exemple, les indicateurs et les mesures pour le tabagisme, l'activité physique et une saine alimentation peuvent être utilisés ensemble ou intégrés à d'autres questions ou encore utilisés comme des modules distincts.

Idéalement, j'aimerais que ces trois éléments soient intégrés aux enquêtes.
Ils permettent de répondre à des préoccupations soulevées par les écoles, soit le fait que l'on délaisse les modèles d'intervention unique. Comme les écoles se tournent vers des stratégies plus complètes pour s'attaquer à un certain nombre de problèmes de santé, nous avons besoin d'un outil qui peut les évaluer de façon appropriée. (R; Prov)

D'autres informations sur les indicateurs et les mesures sont disponibles auprès d'autres sources ${ }^{12}$. L'adoption et l'utilisation de ces indicateurs et de ces mesures vont exiger du temps, mais il s'agit d'une étape essentielle pour assurer la pertinence à la fois scientifique et pratique de la surveillance.

\section{Composante 2 : Échangé de connaissances}

L'échange de connaissances au sein de Youth Excel portait sur des produits et des processus qui faisaient appel à des données de surveillance pour la planification et l'intervention et qui visaient à faciliter l'apprentissage entre pairs quant au renforcement des capacités de DEC.

\section{Transformation des données en produits de connaissance}

Les données issues des systèmes de surveillance doivent être transformées en information afin d'aider à établir une orientation claire à l'égard des nouvelles politiques et des nouveaux programmes, d'évaluer les politiques et les initiatives en place et d'établir des plans stratégiques. La transformation la plus courante mentionnée par les membres de Youth Excel était la transformation des données en rapports. Les rapports sont tous importants pour leur public cible, et ce, quel que soit l'échelon pour lequel ils sont produits : il peut s'agir de rapports provinciaux, locaux ou scolaires, qui fournissent des données comparatives à l'échelle nationale ou provinciale. Les membres ont particulièrement insisté sur la valeur des données à l'échelle locale :

Les intervenants de la province se sont rendu compte à quel point leurs données locales étaient utiles et, au cours de notre dernière table ronde provinciale, ils ont indiqué qu'ils souhaitaient vraiment obtenir des données à l'échelle locale. Ils ne veulent pas mener ne serait-ce qu'une intervention à petite échelle sans disposer de données au niveau de leur école. (R; Prov)

\section{Les bonnes personnes}

Les répondants ont indiqué que les " courtiers du savoir »- les personnes qui transfèrent, rassemblent, interprètent et communiquent l'information - jouent un rôle précieux, en particulier lors des " périodes d'échange des connaissances » qui suivent les périodes de collecte des données (comme les années scolaires).

Nous faisions appel à des courtiers naturels du savoir qui étaient sur place, c'est-à-dire des apprenants en santé, des membres du personnel infirmier en santé publique, des groupes communautaires et d'autres personnes qui étaient en mesure de soumettre les données pour alimenter les discussions sur les interventions. (R; Prov)

\section{Mise en commun et apprentissage au sein} des secteurs et des administrations

Les répondants ont insisté sur les moyens systématiques d'apprendre les uns des autres en vue d'atteindre un objectif commun. Ils ont indiqué accorder une grande importance aux forums organisés par Youth Excel à l'échelle nationale et provinciale. Ils ont affirmé que le dialogue avec d'autres administrations était une source d'inspiration qui aidait les membres de Youth Excel à élaborer leurs idées et à valider leurs résultats.

La table ronde nous a également donné l'occasion d'établir des liens avec des partenaires tels que des employés d'Affaires autochtones et Développement du Nord Canada, ceux des provinces et des territoires, ainsi que d'autres personnes travaillant dans le secteur de l'éducation. La table ronde a été utile, car elle a stimulé la réflexion et a encouragé les partenaires à collaborer pour promouvoir l'adoption d'une approche globale de la santé en milieu scolaire. (P; Nat) 
La continuité des interactions dans le cadre des forums a facilité le maintien des relations à l'échelle nationale :

Le fait de savoir qu'il existe une certaine continuité dans le processus jette les bases et instaure un climat de confiance qui nous permettent de perpétuer les relations et nous aident à réaliser nos projets en tant que consortium et les projets de [Youth Excel] la COALITION sur une base plus régulière. (P; Nat)

Les réunions du réseau provincial, qui avaient lieu au moins deux fois par année, ont permis aux partenaires de chaque province de travailler à l'élaboration d'un programme commun sur le DEC en vue de promouvoir la santé des jeunes. Ces réseaux formels et informels ont constitué des circonstances favorables permettant à plusieurs membres d'exercer leur leadership, de consolider leurs relations et de favoriser leur travail selon un modèle de collaboration non hiérarchique.

Idéalement, les réunions du réseau provincial se tiennent deux fois par année. Après la deuxième table ronde provinciale, nous avons pu constater à quel point les gens appréciaient cet événement. Les réunions du réseau provincial ont commencé à susciter beaucoup d'intérêt. Je recevais des courriels de gens me disant qu'ils en avaient entendu parler et qu'ils souhaitaient y participer. La liste d'invitation a ainsi doublé du jour au lendemain. Nous avons établi un juste équilibre entre les représentants des secteurs de la santé, de l'éducation, de la recherche, des politiques et des pratiques - et même du secteur du tourisme, des parcs et loisirs ainsi que les leaders étudiants. (R; Prov)

Le fait d'associer les forums d'échange des connaissances à une conférence ou un événement connexe (p. ex. une conférence Écoles en santé) a aidé à recruter des participants et à élargir la portée du programme de DEC.

\section{Webinaires}

Les webinaires ont été un moyen de communication efficace pour le DEC et, comparativement aux réunions traditionnelles, ont présenté moins d'obstacles en ce qui concerne l'assiduité. En plus d'éliminer les frais et le temps de déplacement, les webinaires ont pu être enregistrés et mis à la disposition des membres souhaitant y accéder, ce qui a permis d'élargir davantage leur rayonnement.

[Les webinaires] permettent aux gens de chercher et d'obtenir de l'information à jour sur ce qui se passe vraiment. Il s'agit d'un moyen qui permet aux gens de se réunir et de discuter, et qui suscite de l'intérêt et une volonté de collaborer. (R; Prov)

\section{Composante 3 : Compétences et conditions d'utilisation des données probantes}

Les répondants ont considéré les partenariats comme le facteur essentiel au développement des compétences et des conditions d'utilisation des données probantes, ce qui est en cohérence avec les propos portant sur les deux composantes précédentes. Les chercheurs ont besoin que les praticiens et les décideurs accordent de l'importance aux données et qu'ils demandent des données en vue de favoriser la mise en œuvre des interventions et d'orienter les décisions stratégiques. À leur tour, les praticiens et les décideurs ont besoin que les chercheurs continuent de produire des données probantes pertinentes pour favoriser des prises de décision judicieuses. Les chercheurs qui peuvent « trouver des renseignements instantanément » sont particulièrement recherchés, tout comme les " courtiers du savoir » qui savent quelles données sont disponibles et qui sont capables d'analyser et de comprendre les données afin de prendre les mesures appropriées. Même si « dans le passé, les secteurs travaillaient dans un isolement relatif [...], il existe une nouvelle philosophie selon laquelle tous les secteurs doivent collaborer pour atteindre les objectifs de notre programme » (R; Prov).

Les répondants ont souligné qu'il était important de disposer d'un environne- ment favorable à l'utilisation de l'information, qu'il fallait offrir des possibilités de formation pour permettre aux gens d'échanger des connaissances en milieu scolaire et de mettre en œuvre des initiatives utilisant des produits de la connaissance (p. ex. des programmes de subvention scolaires fondés sur les données au niveau de l'école).

\section{Composante 4 : Évaluation}

Les répondants ont reconnu qu'il importait de procéder à une évaluation - pour " tirer des leçons au fur et à mesure » - et que la capacité de produire des données probantes à partir de la pratique était la capacité la moins développée parmi les quatre composantes liées à la capacité de DEC. La capacité de produire l'information la plus utile repose sur la mise en place d'évaluations appropriées. Pour l'ensemble des participants, l'idéal consistait à intégrer les évaluations au système, à utiliser de façon optimale le système de surveillance locale et à assurer la diffusion des conclusions en temps opportun. Les évaluations permettent également d'obtenir un vaste éventail de données probantes, en particulier l'expérience de travail au moyen d'interventions novatrices, les leçons retenues et les pratiques exemplaires parmi les chercheurs, les praticiens et les intervenants des autres administrations.

\section{Capacité globale de DEC}

Quatre thèmes recoupaient les quatre composantes de DEC.

\section{Les partenariats sont essentiels}

La mise à profit des partenariats en cours est un trait spécifique de la capacité de DEC. Ces types de partenariats peuvent varier. Ils comprennent des groupes de recherche universitaires, des ministères et des organismes gouvernementaux ou provinciaux, des agents de la santé publique et des organismes non gouvernementaux :

Les gens qui établiront une relation avec nous et avec qui nous formerons une équipe représentent un autre facteur déterminant. Il peut s'agir de relations avec le sous-ministre et/ou le ministre, les commissions scolaires 
et les membres d'autres équipes de recherche [...] la création de partenariats est essentielle. ( $\mathrm{R}$; Prov)

\section{Vision et leadership stratégique}

Le renforcement de la capacité de DEC exige une vision et un leadership stratégique. Les membres de Youth Excel ont exprimé le besoin d'adopter une vision créée en collaboration et mise à jour régulièrement, vision à laquelle adhérerait l'ensemble des partenaires et des intervenants concernés.

\section{Importance de la terminologie}

De nombreux membres de Youth Excel ont exprimé le besoin d'une terminologie commune. Les termes et les interprétations variaient énormément (p. ex. en approche globale de la santé en milieu scolaire et en application des connaissances) malgré les efforts considérables déployés par les intervenants et les organisations pour s'assurer d'un vocabulaire et de concepts communs.

La communication et l'utilisation d'un langage commun comptent parmi les plus grandes [révélations] [...] et même si notre travail est en grande partie similaire, nous avons tendance à employer des termes différents ou à donner aux mêmes termes des significations différentes. (R; Prov)

En conséquence, il est essentiel de trouver un langage simple, partagé et compris de manière universelle pour communiquer dans le domaine du " renforcement de la capacité de DEC».

Les différences entre le langage et les priorités en matière de recherche par rapport aux politiques et à la pratique représentent un défi; il faudra consacrer du temps et déployer des efforts pour en arriver à un consensus. (R; Prov)

\section{Financement/ressources}

Les répondants ont parlé de l'importance d'obtenir un financement à long terme spécifiquement alloué aux quatre composantes de la capacité de DEC. Les opinions à propos des sources de financement étaient partagées : certains considéraient qu'il était essentiel d'obtenir l'engagement et le financement du gouvernement pour la surveillance et l'évaluation du programme alors que d'autres estimaient qu'il était important d'obtenir une source de financement extérieure au gouvernement pour favoriser la viabilité des initiatives liées à la capacité de DEC (en ce sens qu'une source externe " peut plus facilement surmonter les crises découlant des changements de gouvernement »[R; Prov]). Les répondants ont également souligné la nécessité de faire preuve de créativité à l'égard des sources existantes et des possibilités s'y rattachant.

Nous devrons faire preuve de plus de créativité avec nos ressources actuelles, établir des relations avec nos nouveaux partenaires afin d'élargir notre capacité. Il s'agit d'être plus créatifs dans notre travail. (R; Prov)

\section{Analyse}

\section{Approche axée sur les systèmes}

Nos résultats éclairent les modalités potentielles de renforcement de la capacité de DEC au Canada à partir de l'analyse d'une initiative pancanadienne portant sur les jeunes âgés de 10 à 18 ans. Nous pouvons en dégager cinq observations principales.

Premièrement, le cycle d'amélioration formé des quatre composantes de DEC pourrait aider à orienter les efforts de renforcement de la capacité de DEC. Le modèle provisoire s'est révélé pertinent pour un grand nombre de secteurs et d'administrations, et a aidé les membres de Youth Excel à décrire leur tâche et à demeurer concentrés sur celle-ci, tant sur le plan individuel que collectif.

Deuxièmement, Youth Excel fournit des exemples utiles de transformation du modèle conceptuel de DEC en mesures concrètes. Youth Excel a démontré qu'un système permettant de recueillir et de fournir de la rétroaction, en l'occurrence sur les comportements liés à la santé des jeunes à l'échelle locale (école, communauté), représentait une ressource fondamentale pour l'établissement des mesures prioritaires les concernant. En outre, il est nécessaire de se renseigner sur les interventions innovatrices sur le terrain. Les résultats de ces interventions doivent être documentés de manière à en faciliter la mise en commun, et les détails (tant le « quoi » que le « comment») devraient tout particulièrement être facilement adaptés pour que ces interventions soient utilisables dans d'autres situations. Il faut également mettre en place ume formation régulière et une formation continue. Parmi les mécanismes efficaces, citons les forums d'échange à l'échelon communautaire, provincial/territorial ou national au cours desquels divers types d'intervenants (chercheurs, praticiens et décideurs) provenant de l'ensemble des administrations sont réunis. Ces forums donnent la possibilité de partager des expériences, de former des partenariats, d'établir des liens entre les individus et les groupes et de mettre à contribution les forces et les atouts des intervenants. Les webinaires représentent une autre façon économique de mettre en commun des expériences. Il importe de procéder à des évaluations pour produire de nouvelles données probantes capables d'établir l'efficacité des interventions et de fournir des éléments d'orientation quant à la façon de les améliorer. Les résultats de ces évaluations doivent être synthétisés et mis en commun, de préférence à l'aide de récits descriptifs et mémorables facilement racontables au cours d'un webinaire ou d'un forum tenu à l'échelle provinciale ou nationale.

Troisièmement, l'expérience Youth Excel laisse entrevoir la nécessité d'une approche axée sur les systèmes pour le renforcement de la capacité de DEC. Les résultats ont mis en lumière l'existence d'une série de facteurs interdépendants qui accélèrent ou gênent le renforcement de la capacité de DEC. Parmi ces facteurs, il y a notamment une vision commune, un leadership stratégique, une terminologie commune, un dialogue fréquent, l'existence de partenariats et l'instauration de la confiance. Il s'agit des facteurs les plus étroitement liés aux développements récents des cadres d'application des con- 
naissances*. Dans le domaine de la santé, nous avons en effet assisté, au cours des 15 dernières années, à une évolution des principaux modèles : nous sommes passés de modèles linéaires de transfert des connaissances à des modèles complexes axés sur les relations et les systèmes. Au lieu de mettre l'accent sur les principes et les pratiques d'une médecine fondée sur les preuves (p. ex. la réplication d'interventions précises), on accorde dorénavant plus d'importance aux capacités et aux stratégies organisationnelles ${ }^{12,13}$, à la prise en compte du contexte $^{14,15}$ et au processus d'apprentissage et de changement qui fait intervenir de multiples perspectives (notamment la recherche, les politiques et la pratique) ${ }^{16-21}$. Les modèles de systèmes de transfert des connaissances tiennent compte du fait que les processus de diffusion et de mise en œuvre ainsi que les relations sont façonnés, intégrés et organisés par des structures qui favorisent les interactions entre des intervenants ayant une vision du monde, un langage, des moyens de communication et des attentes qui leurs sont propres ${ }^{22}$. Ces intervenants sont liés entre eux par un système qui, à son tour, est le reflet des cultures, des structures, des priorités et des capacités ${ }^{13}$. Ce système doit être activé pour que ses diverses composantes puissent être reliées afin d'établir des liens entre les connaissances et la pratique 23-25. $^{\text {. }}$ L'activation de ce système pourrait être au cœur de l'initiative Youth Excel.

Quatrièmement, et conformément à l'approche axée sur les systèmes, Youth Excel offre un aperçu des rôles et des relations des partenaires relevant de différentes administrations. Par exemple, les organismes nationaux (CCES et Propel) étaient en meilleure position pour soutenir de façon efficace les efforts au sein d'une province et les efforts interprovinciaux, par rapport à une province qui tenterait d'effectuer ces mêmes activités seule. Citons par exemple le rôle du secrétariat de Propel, la création d'indicateurs et de mesures, l'organisation de forums natio- naux et les programmes d'échange des connaissances avec les pairs. Les intervenants des provinces étaient les mieux placés en renforcement des activités de DEC dans les administrations et entre celles-ci (p. ex. les autorités sanitaires régionales) au sein de leur province. Par exemple, les intervenants provinciaux ont coordonné les enquêtes, ont convoqué les intervenants provinciaux et locaux en vue de la planification des mesures dans leur province et ont adapté les composantes de DEC à leur contexte particulier. Même si les intervenants locaux n'étaient pas membres officiels de Youth Excel, ils ont joué un rôle déterminant dans toutes les activités de DEC; ce sont surtout les interventions locales que les organisations à l'échelle provinciale et nationale permettent de réaliser. L'interaction entre les administrations de Youth Excel fournit des éléments d'information sur la nécessité d'établir un système de DEC comprenant plusieurs paliers et des exemples d'activités susceptible de favoriser la mise en place d'un tel système.

Cinquièmement, l'expérience Youth Excel montre que le renforcement de la capacité de DEC exigera du temps. À la fin de 2011, et donc des trois années de financement de Youth Excel, la capacité de DEC pancanadienne visant à favoriser la santé chez les jeunes était limitée et extrêmement variable entre les diverses administrations. La plupart des développements importants réalisés concernaient des systèmes de surveillance locale et portaient sur l'échange de connaissances, en particulier les rapports de rétroaction destinés à divers publics. On a observé quelques progrès en ce qui concerne l'évaluation, sachant que le processus en avait été amorcé bien avant le début de l'initiative Youth Excel : rappelons qu'au lancement de Youth Excel, cela faisait 16 ans que certains membres collaboraient à divers projets. Les ressources de base, comme la mise au point de systèmes de collecte de données locales et de rétroaction, sont complexes et il faut beaucoup de temps pour les concevoir.

\section{Conclusion}

Le renforcement de la capacité future de DEC axée sur la santé constitue une tâche à la fois stimulante et ardue. Il est stimulant de constater que Youth Excel a été en mesure de mieux faire comprendre les complexités inhérentes à la capacité de DEC et la façon de la renforcer dans divers contextes. Youth Excel a permis de démontrer, grâce à cette expérience, l'importance d'une approche axée sur les systèmes pour traiter du $\mathrm{DEC}^{26}$ et la manière de transformer une approche axée sur les systèmes en ressources et en interventions. Au Canada, nous disposons d'une ressource inestimable : une communauté en pleine croissance qui est résolue et qui s'emploie à établir une plateforme de DEC permettant de réaliser des interventions et des projets spécifiques.

La tâche qui reste à accomplir est ardue, en ce sens que du travail reste à faire et qu'il faut obtenir les engagements connexes nécessaires de la part des multiples administrations (locale, provinciale, territoriale, nationale) et des intervenants (recherche, politiques et pratiques ainsi que santé et éducation). Des travaux antérieurs effectués aux États-Unis (sous la direction de la Society for Prevention Research) ont permis de tirer des conclusions similaires à propos des besoins en matière de systèmes de surveillance communautaire et de rétroaction ainsi que de l'infrastructure à long terme nécessaire pour renforcer ces systèmes. Le fait de répondre à ces besoins au Canada et à l'étranger nous permettra de rendre concrète la vision de Youth Excel, qui consiste à obtenir des données probantes de meilleure qualité et d'en faire une meilleure utilisation au service de la santé des jeunes.

\section{Remerciements}

Les membres de Youth Excel sont PattiJean Naylor et Karen Strange (Université de Victoria); Paul Veugelers et Kate Storey

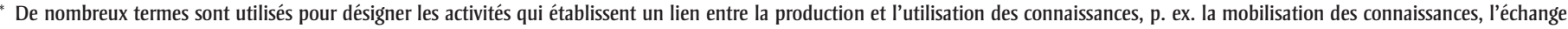
des connaissances et le passage des connaissances à la pratique. Lorsqu'il est question du domaine général, nous utilisons l'expression « application des connaissances », conformément à la définition des $\operatorname{IRSC}^{27}:$ « un processus dynamique et itératif qui englobe la synthèse, la dissémination, l'échange et l'application conforme à l'éthique des connaissances dans le but d'améliorer la santé des Canadiens, d'offrir de meilleurs produits et services de santé et de renforcer le système de santé ».
} 
(Université de l'Alberta); Margaret Schwartz (APPLE Schools); Jane Griffith (Université du Manitoba et Action Cancer Manitoba); Kate McGarry et Tannis Erikson (Action Cancer Manitoba); Heather Manson, Melody Roberts et Jennifer Robertson (Santé publique Ontario); Bill Morrison et Lynn Ann Duffley (Université du Nouveau-Brunswick); Marlien McKay (ministère du Mieux-être, gouvernement du Nouveau-Brunswick); Donna Murnaghan et Brandi Bell (Université de l'Âle-du-Prince-Édouard); Antony Card (Université Memorial de Terre-Neuve [Terre-Neuve-et-Labrador]); Katherine Kelly, Susan Hornby et Jo-Ellen Craig (Consortium conjoint pour les écoles en santé); Steve Manske, Barbara Riley, Christina Kroeker et Katy Wong (Université de Waterloo).

Ce projet de recherche a bénéficié de la contribution financière de Santé Canada, par l'entremise du Partenariat canadien contre le cancer et de la Société canadienne du cancer (subvention $n^{\circ}$ 2011701019). Les promoteurs du projet n'ont cependant participé ni l'élaboration du plan de l'étude, ni à la collecte, à l'analyse ou à l'interprétation des données. Ils n'ont pas pris non plus part à la rédaction de cet article et ne sont pas intervenus dans la décision de le faire publier. Les opinions exprimées sont celles des auteurs et ne correspondent donc pas nécessairement à celles du bailleur de fonds.

\section{Références}

1. Baker Jr. EL, Potter MA, Jones DL, et al. The public health infrastructure and our nation's health. Annu Rev Public Health. 2005;26:303-18.

2. Centers for Disease Control and Prevention. Division of Global Public Health Capacity Development: 2008 Annual Report. Atlanta (GA): U.S. Department of Health and Human Services, Centers for Disease Control and Prevention; 2009.

3. Instituts de recherche en santé du Canada (IRSC). Bâtir une infrastructure durable de recherche en santé publique au Canada. Ottawa (Ont.) : Institut de la santé publique et des populations des IRSC; 2003.
4. Canadian Public Health Association; Provincial and Territorial Public Health Associations. A path toward building public health capacity. Ottawa (Ont) : Canadian Public Health Association and Provincial and Territorial Public Health Associations; 2005.

5. Association canadienne de santé publique au Comité permanent de la santé de la Chambre des communes. Améliorer l'infrastructure des ressources humaines dans le secteur de la santé publique au Canada. Ottawa (Ont.) : ACSP; 2010.

6. Instituts de recherche en santé du Canada (IRSC). L'avenir de la santé publique au Canada : édifier un système de santé publique pour le $21^{\mathrm{e}}$ siècle. Ottawa (Ont.) : IRSC; 2003.

7. Coalition - Connaissances et action liées pour une meilleure prévention [Internet]. Toronto (Ont.) : Partenariat canadien contre le cancer; 2012 [consulté le 6 novembre 2012]. Accessible à l'adresse suivante: http://www.partnershipagainstcancer.ca/fr /priorities/2007-2012-initiatives/primary -prevention-2007-2012-strategic-initiatives /coalitions-linking-action-science-for-prevention -clasp-2/

8. White House Task Force on Childhood Obesity. Solving the problem of childhood obesity within a generation: White House Task Force on Childhood Obesity Report to the President. Washington (DC) : Executive Office of the President of the United States; 2010.

9. Lagarde F, LeBlanc CM, McKenna M et collab. Cadre pour une politique scolaire : mise en œuvre de la stratégie de l'OMS pour l'alimentation, l'exercice physique et la santé. Genève $(\mathrm{CH})$ : Organisation mondiale de la santé; 2008.

10. Riley BL, Manske S, Cameron R. Youth Excel: towards a pan-Canadian platform linking evidence and action for prevention. Cancer. 2011;117(10 Suppl):2281-8. doi: $10.1002 /$ cncr. 26046 .

11. Agence de la santé publique du Canada. Freiner l'obésité juvénile : Cadre d'action fédéral, provincial et territorial pour la promotion du poids santé. Ottawa (Ont.) : Agence de la santé publique du Canada; 2012.
12. Frenk J. Balancing relevance and excellence: organizational responses to link research with decision making. Soc Sci Med. 1992;35(11):1397-404.

13. Best A, Terpstra JL, Moor G, Riley B, Norman CD, Glasgow RE. Building knowledge integration systems for evidenceinformed decisions. J Health Organ Manag. 2009;23(6):627-41.

14. Green LW. From research to 'best practices' in other settings and populations. Am J Health Behav. 2001;25(3):165-78.

15. Van de Ven AH, Johnson PE. Knowledge for theory and practice. Acad Manag Rev. 2006:31(4):802-21.

16. Rychetnik L, Frommer M, Hawe P, Shiell A. Criteria for evaluating evidence on public health interventions. J Epidemiol Community Health. 2002;56(2):119-27.

17. Best A, Stokols D, Green LW, Leischow S, Holmes B, Buchholz K. An integrative framework for community partnering to translate theory into effective health promotion strategy. Am J Health Promot. 2003;18(2):168-76.

18. Green LW. Public health asks of systems science: to advance our evidence-based practice, can you help us get more practice-based evidence? Am J Public Health. 2006;96(3):406-9. doi: 10.2105/AJPH.2005 .066035 .

19. Glasgow RE, Emmons KM. How can we increase translation of research into practice? Types of evidence needed. Annu Rev Publ Health. 2007;28:413-33. doi: 10.1146 /annurev.publhealth.28.021406.144145.

20. Green LW, Glasgow RE, Atkins D, Stange K. Making evidence from research more relevant, useful, and actionable in policy, program planning, and practice slips 'twixt cup and lip’. Am J Prev Med. 2009;37 (6 Suppl 1):S187-91. doi: 10.1016/j.amepre.2009.08 .017 .

21. Green LW, Ottoson JM, García C, Hiatt RA. Diffusion theory and knowledge dissemination, utilization, and integration in public health. Annu Rev Publ Health. 2009;30:15174. doi: 10.1146/annurev.publhealth.031308 .100049 . 
22. Frenk J. Balancing relevance and excellence: organizational responses to link research with decision making. Soc Sci Med. 1992;35(11):1397-404.

23. Best A, Holmes B. Systems thinking, knowledge and action: towards better models and methods. Evidence \& Policy. 2010:6(2):14559. doi: 10.1332/174426410X502284.

24. Best A, Hiatt RA, Norman CD; National Cancer Institute of Canada Joint Working Group on Translational Research and Knowledge Integration of the Advisory Committee for Research and the Joint Advisory Committee for Cancer Control. Knowledge integration: conceptualizing communications in cancer control systems. Patient Educ Couns. 2008;71(3):319-27. doi: 10.1016/j.pec.2008.02.013.

25. Holmes BJ, Finegood DT, Riley BL, Best A. Systems Thinking in dissemination and implementation research. Section 2. Dans : Brownson RC, Colditz GA, Proctor EK (dir.). Dissemination and Implementation Research in Health: Translating Science to Practice. Oxford: Oxford University Press; 2012. 175191.

26. Agence de la santé publique du Canada. Freiner l'obésité juvénile : Cadre d'action fédéral, provincial et territorial pour la promotion du poids santé. Ottawa (Ont.) : Agence de la santé publique du Canada; 2012.

27. Instituts de recherche en santé du Canada (IRSC). À propos de l'application des connaissances aux IRSC [Internet]. Ottawa (Ont.) : IRSC; 2003 [consulté le 11 décembre 2013]. Consultable à la page : http:// www.cihr-irsc.gc.ca/f/39033.html 